\title{
Kulturlandschaftswandel in der Schweiz (KLW-Programm)
}

\author{
Kartierungslegenden \\ und vereinfachte erläuternde Beispiele
}

\author{
1. Nutzflächenkartierung \\ 2. Grundbesitzkartierung \\ 3. Gebäudenutzungskartierung \\ 4. Gebäudekonstruktionskartierung \\ 5. Gebäudemutationskartierung
}

Bemerkung: In den erläuternden Kartierungsbeispielen mußten wegen der beträchtlichen Verkleinerung aus graphischen Gründen die Parzellengrenzen weggelassen werden. Zur Illustration wird in
Fig. 3 (Breno TI) für einen kleinen Ausschnitt das Parzellenmuster aufgezeichnet. Die Kartierung stützt sich selbstverständlich auf die Einheit der Besitzparzelle. 
$\mathrm{Nutz}$ f 1 äche $\mathrm{c}-\mathrm{K}$ artie rung nachgeführt 24.9. 74

Nutzflächen ausserhalb der Haushofstätten (ohne Hausgärten etc。)

+ * bedeutet : zusätzliche Schwarzsignatur

( ) bedeutet : Signatur in der entspr. Farbe

\section{Getreide : $\quad$ Prismalo $\mathrm{Nr}_{0}+$ *}

Weizen . . . . . . . . . 10

Gerste, Hafer ...... . . 35

Roggen ............. $10+$ (. 17

Dinkel . . . . . . . . . . . $10+[\because \because 9$

Mischel von Brotgetreiden

Roggen und Weizen . . . . . (10

Mischel von Futtergetreiden

Gerste und Hafer . . . . . . (35

Futter:

Kunstwiese, Kunstfutteranbau . . 210

Naturwiese Fettmatten . . . . 245+

$$
\text { Magermatten . . . } 245
$$

Naturwiese mit Obst . . . . . . 245+ ○О잉

Kastanienselve . . . . . . . $245+8$

Weide

$$
\begin{aligned}
& \text { Dauerweide . . . . } 249 \\
& \text { Koppelweide. . . } 249+ \\
& \text { Waldweide . . . . }(249 \text { ( . . (249 } \\
& \text { Mähweide . . }
\end{aligned}
$$

Streuland . . . . . . . . . (249 VIII/ $)$

Kunstwiese im Uebergang

zur Naturwiese..... . (210/245

Hackfrüchte:

Kartoffeln . . . . . . . . . 59

Saatkartoffeln . . . . . . . . (59

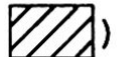

Runkelrüben . . . . . . . 81

Zuckerrüben . . . . . . . . . . $81+8 \because \because$

Obst, Beeren und Gemüse:

Reine Obstkultur ...... (70 (\%)
Reben . . . . . . . . . 70

Beeren . . . . . . . . 80

Feldgemüse . . . . . . . . . 110

Südalpine Mehrfachkultur . . . (70/80 Vi\%)

Weitere Kulturen :

Mais .. . . . . . . 50

Raps .............. $50+$ PD

Tabak ............... $50+\mathrm{VVV}$

Acker- und Sojabohnen .. . . . 50+ $50+000$

Gairtenland :

Gärtnerei, Garten . . . . . . 161

Gartenbaumschule . . . . . 160

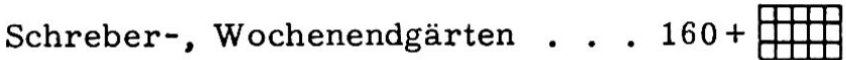

Unterglas (Plastic) Kultur . . . . 161+

Wald, Hecken :

Aufforstung . . . . . . . . (229

Baumschule . . . . . . . (229 [\%०)

"Verwaldung" (wild) . . . . . . 229+

Strauchhecke, Ufergehölz . . . . (229 $\times \times \times \times$ )
(mind. $50 \mathrm{~m}$ lang)

Baumhecke (mind。 $50 \mathrm{~m}$ lang) . . (229 $\rightarrow$ )

Kleingehölz, Baumgruppe . • . . (229 $::::$ )

Freiland:

Verödetes Wirtschaftsland (Brache) . 5

Natürliches Oedland (unproduktiv) . . 5 nur um-

Riedland (ungenutzt) . . . . . (5 E-

Weitere Signaturen:mit Bleistift (schwarz)

Schuttdeponie . . . . . . . .

stark vernachlässigte Kultur

(verunkrautet, schädlingsbefallen u. ä.)

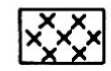

Abbaugebiete (soweit nicht in Karte vorgedruckt) Kiesgruben, Steinbrüche etc. 
Fig. 2: Villars-sur-Glâne FR, 1972: Ausschnitt aus der Nutzflächenkartierung von U. Schnydrig (Geographisches Institut der Universität Fribourg).

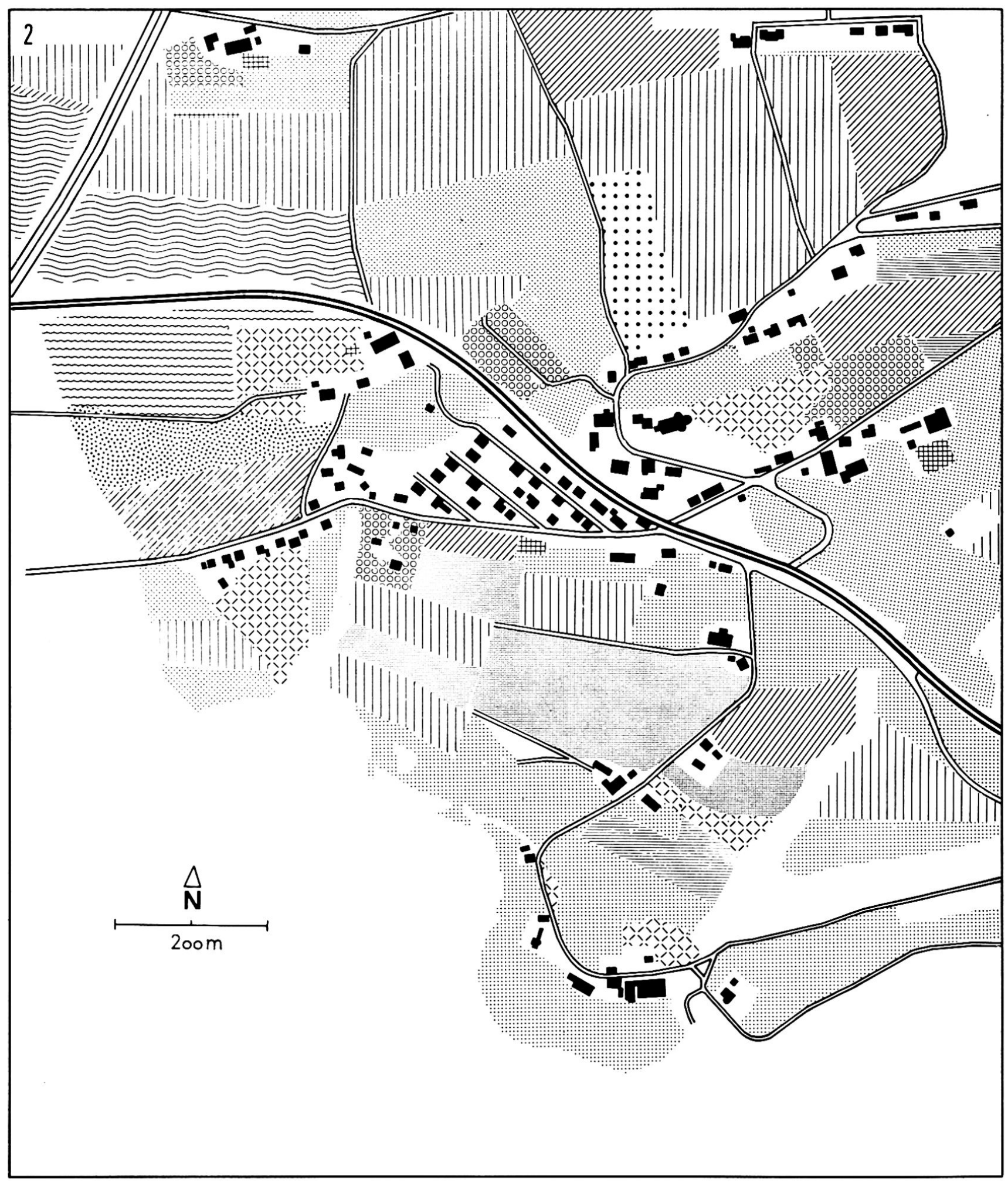


Fig. 3: Breno TI, 1972: Ausschnitt aus der Nutzflächenkartierung von Dr. W. Leimgruber.

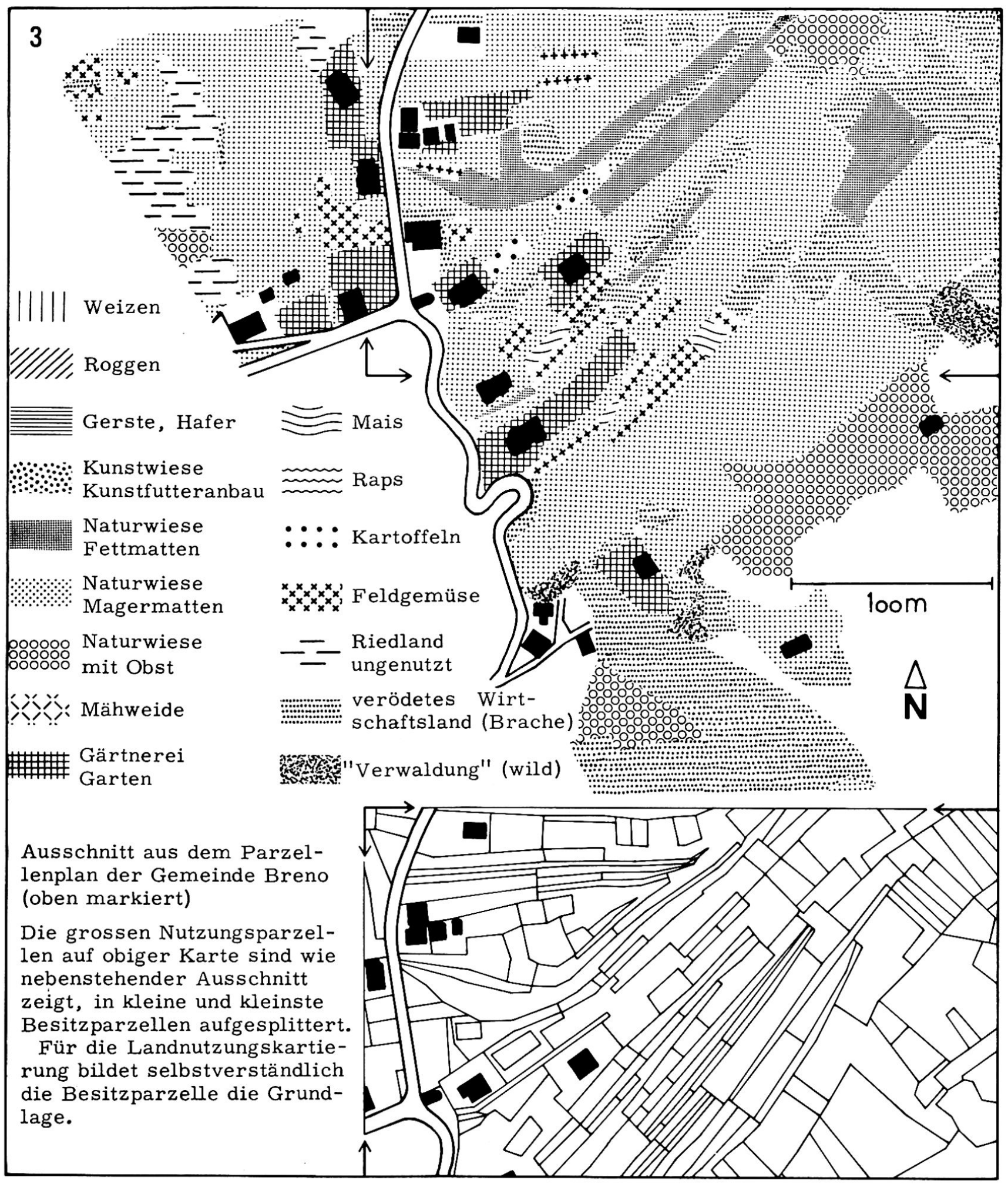


Fig. 4: Urmein GR, 1973: Grundbesitzkartierung von Frau Ch. Stocker.

Arbeitsgrundlage: Güterzusammenlegungsplan FlerdenUrmein 1:5000 (Provisorische Neuzuteilung Stand April 1964). Reproduziert mit Bewilligung des Meliorations- und Vermessungsamtes Graubünden, Chur v. 18. 2. 1974.

Grund b e s itz-Kartie rung nachgeführt 26.2.74

1. Berufliche und juristische Stellung des Grundbesitzers

Grundfarbe : Farbnummer PRISMALO

1. Vollberufliche Landwirte . 210 dunkelgrün

2. Nebenberufliche Landwirte - 245 oliv

3. Landwirtschaftliche Rentner und Erbengemeinschaften . 230 hellgrün

4. Nicht-landwirtschaftliche Rentner und Erbengemeinschaften . . 5 grau

5. Natürliche Personen im sekundären Wirtschaftssektor tätig . . 70 rot

6. Juristische Personen des sekundären Wirtschaftssektors . . . 110 violett

7. Natürliche Personen im tertiären Wirtschaftssektor tätig. . 161 hellblau

8. Juristische Personen des tertiären Wirtschaftssektors . . . 160 dunkelblau

9. Eidgenossenschaft, Kantone 59 braun
10. Einwohnergemeinden. . . 240 gelb

11. Uebrige Gemeinden, sowie Korporationen und Stiftungen öffentlichen und privaten Rechts . . . . . 50 orange

2. Steuerdomizil des Grundbesitzers (Ohne Positionen 1.9, 1.10 und 1.11 und ohne Verkehrswege)

Schraffur :

1. In der Testgemeinde . . (keine)

2. Im gleichen Kanton . . . horizontal, weit

3. In der übrigen Schweiz . . vertikal, eng

4. Im Ausland . . . . . kreuzweise, eng

3. Staatszugehörigkeit des Grundbesitzers (Natürliche Personen)

lo Schweiz . . . . . . keine Punktierung

2. Ausland . . . . . . punktiert

4. Grenzen

1. Grenze der Bauzone . . schwarz

2. Gemeindegrenze. . . schwarz gestrichelt

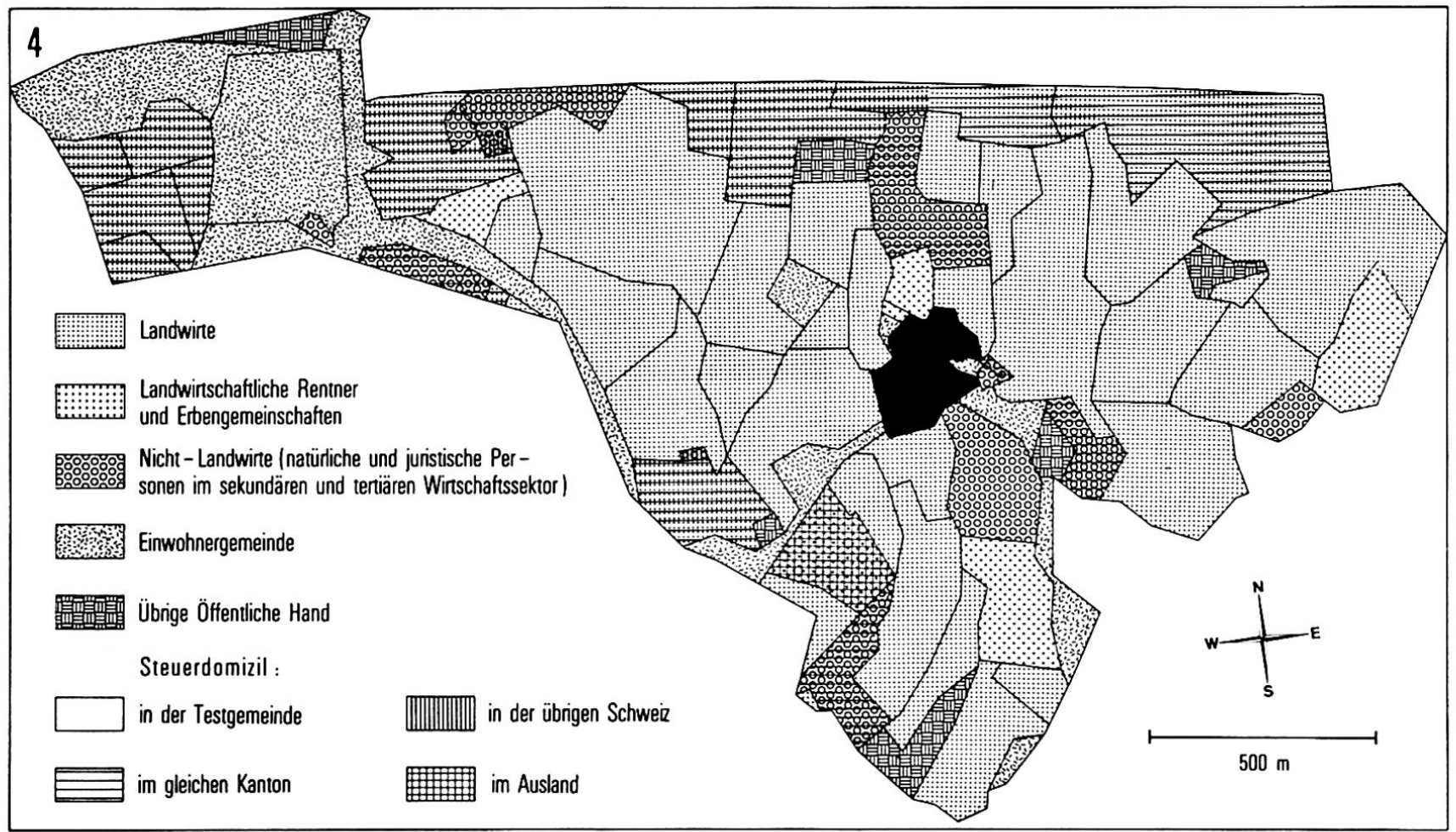


G e bäudenutzung $s-K a r t i$ e $r u n g$ 12. 7.72

\section{Gebäudenutzung}

dauernd bewohnt

periodisch bewohnt, landwirtschaftl. . periodisch bewohnt, nicht landw. . . ungenutzt . . . . . . . . . . öffentliche Gebäude • • • • • • • . Verwaltung Schule PTT<smiles>[CH]1[CH][CH]C[CH]1</smiles>

Spital • • • • • . • . . .

Sanatorium . . . . . . .

Altersheim

Oeffentliche Gebäude anschreiben oder mit Verzeichnis numerieren!

Kirche . . . . . . . . . . . +

Kirchgemeindehaus ....... . 4

Pfarrhaus . . . . . . . . .

Gewerbe, Geschäft . . . . . •

Gastgewerbe . . . . . . .

Restaurant in Wohnhaus . . . . .

Hotel, Motel

Stall

Schweinestallungen ....... 66666

Hühnerstallungen . . . . . . 44444

Speicher, Scheune für Getreide und andere Ackerprodukte

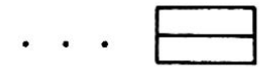

Heuscheune . . . . . . . . . .

Schuppen, Garage . . . . . . . $\square$

Industrie

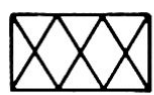

Gebäudenutzung, Beispiele

für Kombinationen

Wohnhaus über Stall . . . . . . .

Wohnhaus mit angebautem Stall

Wohnhaus mit Stall, Heu - und Getreidescheune . . $\$(\otimes)$ b b nebeneinander mit Einfahrt

Alpstall mit Sommerwohnteil

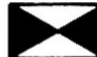

Gewerbe oder Geschäft in Wohnhaus

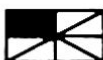

Gewerbe und Gasthaus (z. B. Gasthaus mit Metzgerei) sofern nicht nebeneinander (bewohnt)

Bauernhaus mit Stall und Ferienwohnung

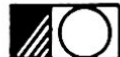

Gewerbezweige durch Zahlenlegenden angeben!

Kleinsignaturen

Brunnen .. . . . . . . . . . .

Miststock . . . . . . . . . $\times$

Silo . . . . . . . . . . . .

Bienenhaus . . . . . . . . . $\triangle$

Trafo-Station . . . . . . . . \ 
Fig. 5: Gelterfingen BE, 1973: Ausschnitt aus der Gebäudekartierung von PD Dr. K. Aerni (Geographisches Institut der Universität Bern).

Arbeitsgrundlage: Übersichtsplan der Gemeinde Gelter-

fingen. Reproduktion mit Bewilligung der Eidg. Vermes-

sungsdirektion vom 20. 2. 1974).

Gebäudenutzung.
Fig. 6: Gelterfingen BE, 1973: Dachformen und Dachbedeckung.
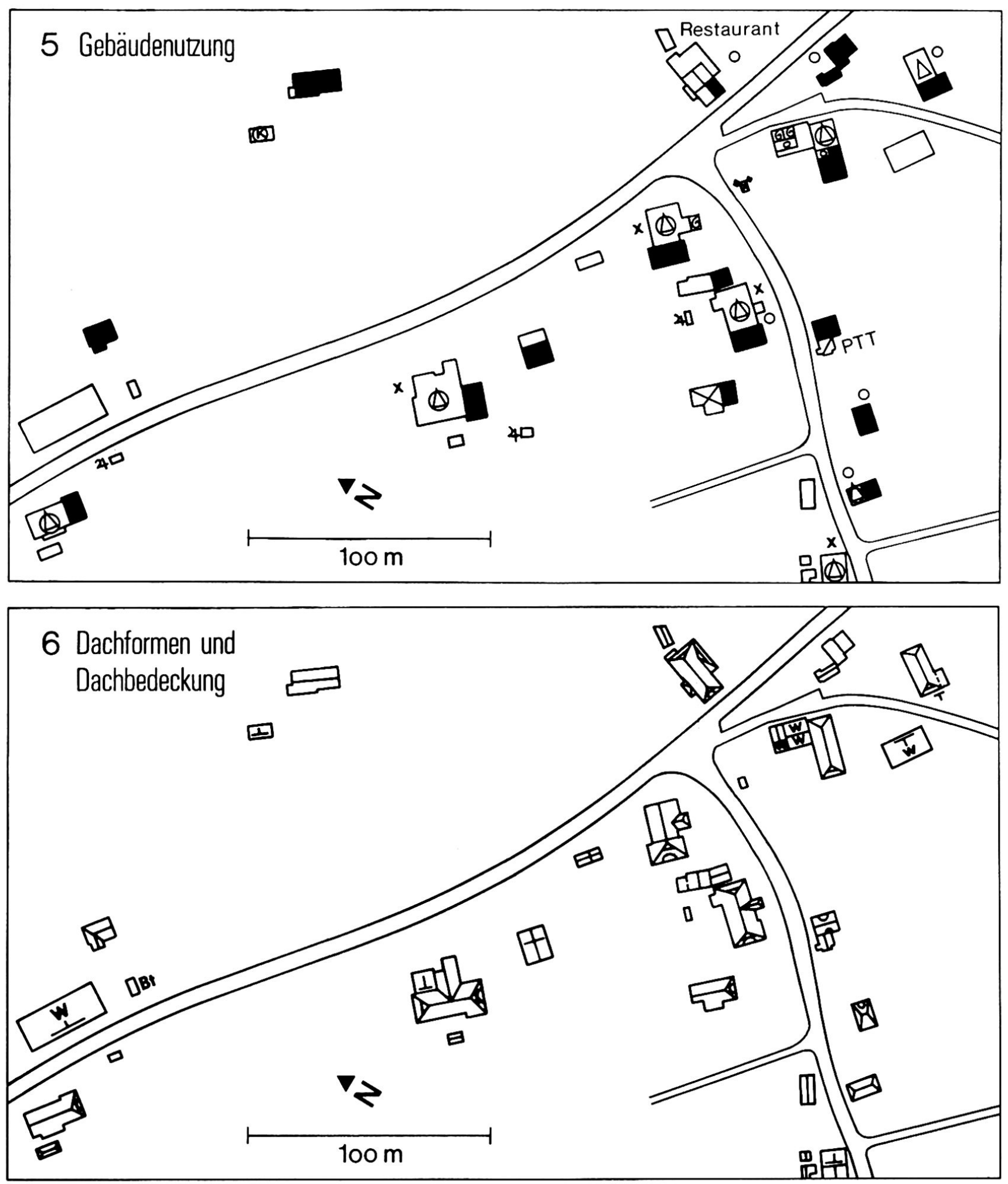
Fig. 7: Gelterfingen BE, 1973: Wandkonstruktion.

Fig. 8: Gelterfingen BE, 1973: Gebäudemutationen.
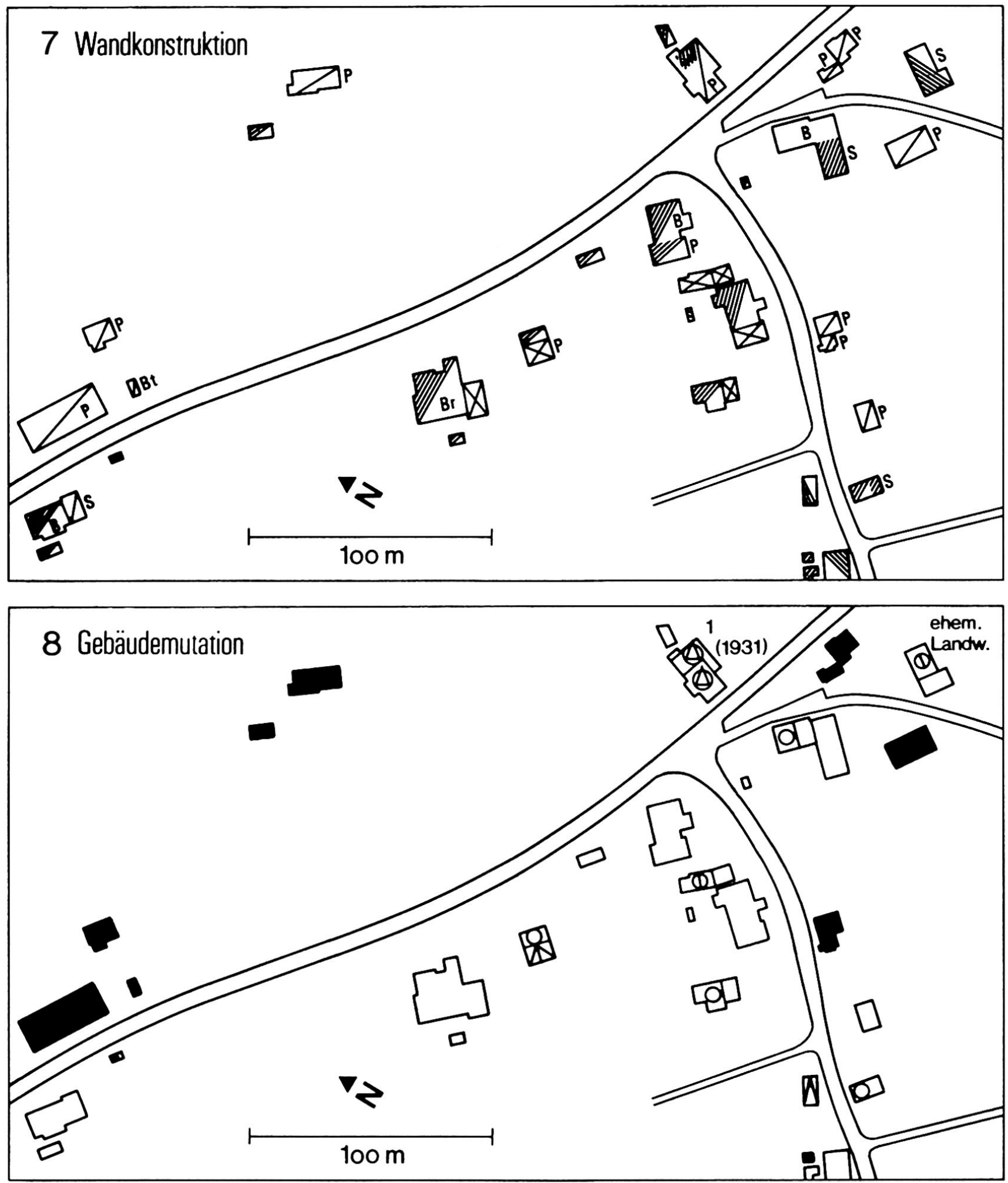
öffentliche Waage . . . . . . . .

Feuerwehrmagazin . . . . . . . . $\times$

Farben : Ganze zum Gebäude gehörige Parzelle kolorieren :

Landwirtschaft . . . grün . Prismalo 245

öffentliche Gebäude . • blau . . ." . 161

Gewerbe, Geschäft,

Gastgewerbe . . . . rosa . . ". 81

periodischer Wohnbau

nicht landwirtschaftlich gelb . . ". 10 (Ferienhäuser)

Industrie . . . . violett . . . . 110

übriger Wohnbau . keine Farbe für Parzelle

Landwirts chaftlicher

Wohnbau .... . grün !

Gebäudekonstruktions-Kartierung nachgeführt 27.2.74

a. Dachformen

Vollwalm, Hauptfensterfront. . . .

Halbwalm

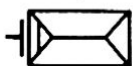

Gerschild

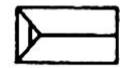

Rundgiebel

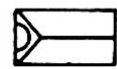

Kreuzfirst mit Ründi

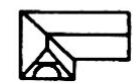

Satteldach

Satteldach mit Knick

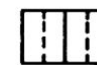

Mansardendach

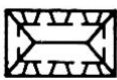

Pyramidendach

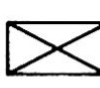

Pultdach

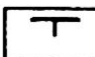

Klebdächlein . . . . . . . . . U U

Flachdach . . . . . . . . . . $\square$

b. Dachbedeckung

Ziegel allgemein,

Falz- und Pfannziegel • • • • • •

Biberschwänze . . . . . . . .

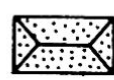

Schindeln

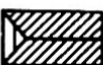

Strohdach

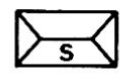

Steinplatten

Schiefer

\section{Eternitschiefer}

(Schraffur quer zu First)

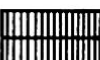

Welleternit . . . . . . . . . . W

Blechdach •. . . . . . . . . B

Kupferblechdach .......... .

Glasdach . . . . . . . . . . G

c. Wandkonstruktion

Steinbauten :. . . . . . . .

Bruchstein . . . . . . . .

Backstein . . . . . . . . .

Beton . . . . . . . . . . . . Bt

Haustein . . . . . . . . . . H

Kunststein . . . . . . . . . . . K

Elemente . . . . . . . . . . E 
Fachwerkbauten : . . . . . .

Holzbauten :

Rundholz-Block ...........

Hälbling-Block • . . . . . . .

Kantholz-Block . . . . . . . .

Ständerbau

Bretter, verschiedene

Holzkonstruktionen

Verputzte und

verschalte Bauten: $\cdot$. $\cdot$.

Putz . . . . . . . . . .

Schindeln und Schipfen.

Blech . . . . . . . . . . .

Eternit . . . . . . . . .

Mantel (Innen Blockbau, aussen Putzmantel) (Engadin)
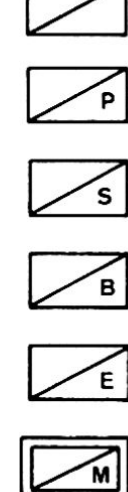

Baten aus modernen Materialien :

Stahl- und Holzkonstruktionen mit Glas

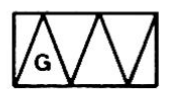

Stahl- und Holzkonstruktionen mit Eternit

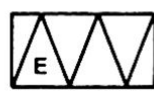

Stahl - und Holzkonstruktionen mit Buntmetall- oder Leichtmetallblech

Stahl- und Holzkonstruktionen mit Kunststoff

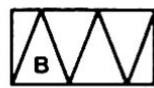

Beton-Skelettbau . . . . . . . . НHД
Gebäudemutations-Kartierung 12.7. 72

Farben für ursprünglich

landwirtschaftliche Bauten . . . . grün

Farben für ursprüngliche Wohnbauten . rot

Farben für ursprünglich andere Bauten blau

ganz: partiell:

Funktionswechsel...... $\bigcirc$

Renovation . . . . . . . . $\triangle$

Aufstockung . . . . . . . . $\square$

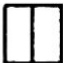

Neubau an derselben Stelle, .. ohne Funktionswechsel

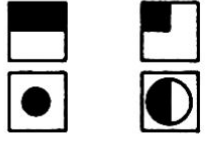

Funktionswechsel mit Renovation $\theta$

Funktionswechsel mit .....

Gebäudeerhöhung

Renovation und Erhöhung

$\otimes$

Neubau, ohne Vorgänger . . .
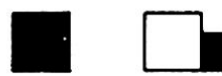

Camping, neu angelegt ...

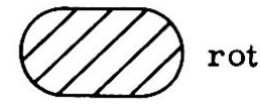

Camping, aufgehoben . . .

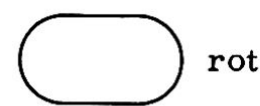

Platz mit permanenten

Wohnwagen, neu rot

Platz mit permanenten

Wohnwagen, aufgehoben ${ }^{\bullet} \cdot$

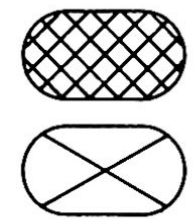
rot 